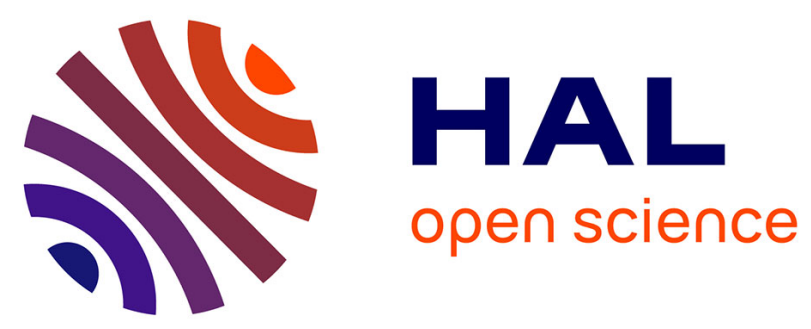

\title{
Euroscepticisme, populisme, droites radicales : état des forces et enjeux européens
}

\author{
Gilles Ivaldi
}

\section{To cite this version:}

Gilles Ivaldi. Euroscepticisme, populisme, droites radicales: état des forces et enjeux européens. L'Europe en formation. Revue d'études sur la construction européenne et le fédéralisme, 2014, Euroscepticisme et élections européennes de 2014, 2014/3 (373), pp.7-28. 10.3917/eufor.373.0007 . halshs-01385790

\section{HAL Id: halshs-01385790 \\ https://shs.hal.science/halshs-01385790}

Submitted on 23 Feb 2018

HAL is a multi-disciplinary open access archive for the deposit and dissemination of scientific research documents, whether they are published or not. The documents may come from teaching and research institutions in France or abroad, or from public or private research centers.
L'archive ouverte pluridisciplinaire HAL, est destinée au dépôt et à la diffusion de documents scientifiques de niveau recherche, publiés ou non, émanant des établissements d'enseignement et de recherche français ou étrangers, des laboratoires publics ou privés. 


\section{EUROSCEPTICISME, POPULISME, DROITES RADICALES : ÉTAT DES FORCES ET ENJEUX EUROPÉENS}

Gilles Ivaldi

Centre international de formation européenne | «L'Europe en Formation »

$2014 / 3 n^{\circ} 373$ | pages 7 à 28

ISSN 0014-2808

ISBN 9770014280200

Article disponible en ligne à l'adresse :

https://www.cairn.info/revue-l-europe-en-formation-2014-3-page-7.htm

\section{Pour citer cet article :}

Gilles Ivaldi, «Euroscepticisme, populisme, droites radicales : état des forces et enjeux européens », L'Europe en Formation 2014/3 (n³73), p. 7-28.

DOI 10.3917/eufor.373.0007

Distribution électronique Cairn.info pour Centre international de formation européenne.

(C) Centre international de formation européenne. Tous droits réservés pour tous pays.

La reproduction ou représentation de cet article, notamment par photocopie, n'est autorisée que dans les limites des conditions générales d'utilisation du site ou, le cas échéant, des conditions générales de la licence souscrite par votre établissement. Toute autre reproduction ou représentation, en tout ou partie, sous quelque forme et de quelque manière que ce soit, est interdite sauf accord préalable et écrit de l'éditeur, en dehors des cas prévus par la législation en vigueur en France. Il est précisé que son stockage dans une base de données est également interdit. 


\section{Euroscepticisme, populisme, droites radicales: état des forces et enjeux européens}

\section{Gilles Ivaldi}

Gilles Ivaldi est Chargé de recherche CNRS en science politique à l'URMIS (Université de Nice). Ses travaux portent sur les changements des systèmes partisans et l'étude des partis de droite radicale en France et en Europe. Il a notamment publié Droites Populistes et Extrêmes en Europe occidentale (Paris: La Documentation française, 2004) et The 2012 French Presidential Elections. The Inevitable Alternation (Palgrave MacMillan, 2013), en collaboration avec Jocelyn Evans.

\section{Introduction}

Les élections européennes des 22 au 25 mai 2014 ont été dominées par la poussée des forces eurosceptiques. Cette percée, nourrie par le rejet des élites communautaires, sur fond de crise économique et de politiques d'austérité, a propulsé sur le devant de la scène politique européenne des formations situées, pour la plupart d'entre elles, à la périphérie des systèmes partisans. Très largement anticipé par les enquêtes d'opinion, ce mouvement de crispation a alimenté nombre de spéculations quant à une possible alliance des populistes européens, avec en ligne de mire un éventuel blocage du fonctionnement institutionnel de l'Union. Dès les premiers mois de 2014, les leaders européens, par la voix de José Manuel Barroso, Martin Schultz ou Herman van Rompuy notamment, ont fait état de leurs inquiétudes face à une telle menace, reconnaissant les difficultés de l'UE à maintenir le lien avec ses citoyens et invitant les électeurs à se détourner des démagogues et autres tribuns populistes.

La lecture des résultats du scrutin de mai dernier et la mesure effective de l'ampleur de la "vague " eurosceptique paneuropéenne s'avèrent toutefois problématiques. Un premier ordre de difficulté tient aux possibles effets de prisme qui résultent de l'agrégation de scrutins nationaux, du poids différentiel des divers États membres et du risque, sans doute, de traduire au niveau européen des 
dynamiques liées, pour partie au moins, à l'état des rapports de forces politiques nationaux. Le rejet de l'Europe demeure en second lieu un phénomène très hétérogène, recoupant des réalités partisanes diverses ainsi que de multiples axes de conflits. Les dimensions économique, politique et culturelle, sur lesquelles s'articulent les dynamiques d'opposition à l'UE, constituent autant de lignes de clivages qui traversent aujourd'hui l'espace public européen. Hors de tel ou tel contexte national spécifique, l'approche comparative illustre les limites de l'usage médiatique de termes polémiques tels que "populisme " ou " euroscepticisme". Ces notions méritent d'être mieux définies, leur périmètre mieux délimité, pour isoler notamment les acteurs politiques qui représentent aujourd'hui le véritable défi pour l'Union européenne, parce que porteurs de projets fondamentalement antagonistes à ses principes et à ses valeurs.

\section{De l'hétérogénéité des forces d'opposition à l'Europe}

Les élections de mai 2014 ont témoigné d'une accentuation de la fragmentation du système de partis transnational et du déclin des acteurs " mainstream " dépositaires historiquement du projet européen. Ces derniers - conservateurs, sociaux-démocrates, libéraux et écologistes - réunissaient encore plus de $85 \%$ des sièges au Parlement il y a vingt ans, contre $70 \%$ aujourd'hui. Ce recul des grands acteurs de gouvernement agit comme un révélateur des difficultés de l'Union européenne: crise économique et sociale, évidemment, mais au-delà discrédit politique nourri par le déficit démocratique chronique dont souffrent les grandes institutions de l'UE.

Sur fond d'abstention massive, les résultats du scrutin ont posé une nouvelle fois la question fondamentale de la représentation et du linkage, c'est-à-dire la nature du lien qui rattache les citoyens européens aux élites politico-administratives de l'Union, quand bien même l'adhésion à la construction européenne demeure majoritaire dans la plupart des États membres. La crise de l'UE est d'abord une crise de confiance: aujourd'hui, moins d'un tiers (31\%) des Européens disent avoir confiance en l'Union, une érosion de près de 17 points depuis $2008^{1}$. Le sentiment d'un "disempowerment", d'une perte de contrôle et d'une déconnexion croissante d'avec une bureaucratie européenne coupée de ses citoyens, est dominant. $^{2}$

1. Eurobaromètre standard n 81, réalisé du 31 mai au 14 juin 2014 (EU 28, N=28 004 répondants).

2. Interrogés dans sept des principaux États membres à la veille du scrutin, $71 \%$ des citoyens déclaraient que "leur voix ne compte pas dans l'UE ", $65 \%$ estimaient que cette dernière " ne comprend pas les besoins de ses citoyens ", $63 \%$ qu'elle est " intrusive " et $57 \%$ qu'elle est " inefficace ». Cf. Pew Research Center, Spring 2014 Global Attitudes Survey, sondage réalisé sur des échantillons nationaux de 1.000 répondants en France, Allemagne, Grèce, Italie, Pologne, Espagne et Grande-Bretagne (http://www.pewglobal.org/2014/05/12/a-fragile-rebound-for-eu-image-on-eve-of-european-parliament-elections/). 
Indéniablement, les formations eurosceptiques ont été en mesure de jouer sur de tels sentiments de défiance, attaquant de front la «bureaucratie " et les élites bruxelloises. En mai dernier, ces forces anti-européennes - au sens le plus large - distribuées dans les groupes des Conservateurs et Réformistes européens (CRE), Europe de la Liberté et de la Démocratie directe (ELDD) et parmi les non-inscrits ont obtenu un total de 170 élus, soit près de $23 \%$ des sièges au Parlement de Strasbourg, soit une progression au regard des $16 \%$ totalisés en 2009 (Cf. Figure 1).

Figure 1. Résultats des partis eurosceptiques au Parlement européen

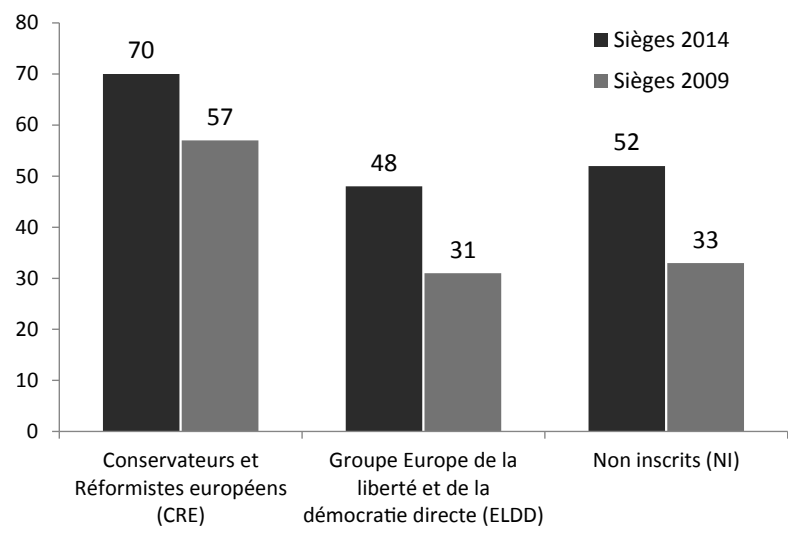

Résultats en nombre de sièges

La nature de cette mobilisation par les forces anti-européennes demeure toutefois profondément hétérogène, tant en termes des types d'acteurs représentant la grande "famille " eurosceptique, qu'au regard des enjeux sur lesquels le succès électoral de ces partis s'est appuyé. La littérature consacrée au phénomène eurosceptique permet d'identifier, au minimum, deux éléments de distinction importants.

Le premier renvoie à l'intensité de l'opposition à l'Europe et, indirectement, à la position «systémique » des mouvements qui en sont porteurs. Certains auteurs proposent notamment de distinguer entre un euroscepticisme "soft " et une forme plus dure $(\text { hard })^{3}$, nous rappelant que de tels partis se rencontrent à la périphérie mais aussi au centre des systèmes partisans, tels le Parti démocratique civique (ODS) tchèque, l'Alliance néo-flamande (N-VA) ou l'Alliance Nationale

3. Cf. Szczerbiak, Aleks et Paul Taggart, "Theorising Party-Based Euroscepticism: Problems of Definition, Measurement and Causality "In: Comparative and Theoretical Perspectives. Opposing Europe? The Comparative Party Politics of Euroscepticism, 2, (Oxford: Oxford University Press 2008), pp. 238-262. 
lettone $(\mathrm{TB} / \mathrm{LNNK})^{4}$. Ces derniers sont alliés, notamment, aux conservateurs britanniques de David Cameron ou aux Irlandais du Fianna Fáil, qui incarnent parfaitement ce courant de l'antifédéralisme "mainstream" au sein du groupe des Conservateurs et Réformistes européens (CRE). Ici, l'Euroscepticisme - appréhendé parfois en termes "d'euro-réalisme »-porte non pas tant sur le projet d'intégration européenne que sur la trajectoire actuelle ou future de la construction de l'UE, au regard singulièrement des questions de transferts supplémentaires de compétences ou d'élargissement.

S'agissant des eurosceptiques "durs ", en revanche, nous sommes en présence de partis opposés par principe à l'intégration et à l'abandon de souveraineté nationale, jusqu'à réclamer, pour les plus jusqu'au-boutistes d'entre eux, la sortie pure et simple de l'Union européenne. Ici, un second élément de différenciation s'établit en fonction de la position spatiale de ces mouvements. En termes partisans, en effet, cet euroscepticisme balaie un spectre large de formations distribuées aussi bien à droite qu'à gauche du spectre politique, quand bien même l'opposition la plus résolue à l'Europe conserve un fort tropisme droitier : sur une soixantaine de partis eurosceptiques présents lors des élections de 2014, plus des trois quarts sont orientés à droite'. À gauche de l'échiquier, cet euroscepticisme est visible dans les positions de partis tels que l'AKEL chypriote, le parti communiste de Bohême et de Moravie en République Tchèque, le parti communiste grec (KKE), le Parti socialiste (SP) aux Pays-Bas ou les partis écologistes (MP) et de Gauche (V) en Suède. L'hostilité à l'Europe est également centrale dans la doctrine hétéroclite du populisme "vert » incarné par le Movimento 5 Stelle (M5S) de Beppe Grillo chez nos voisins italiens. Souvent, les observateurs ont ajouté à tort à cette catégorie eurosceptique certains mouvements de gauche critique, telles la Coalition de la Gauche radicale (SYRIZA) d'Alexis Tsipras en Grèce, Die Linke en Allemagne ou la Gauche Plurielle (IP) espagnole de Willy Meyer, réunis au sein du groupe de Gauche unitaire européenne/Gauche verte nordique (GUE/NGL). Si ces formations se montrent effectivement particulièrement critiques à l'encontre des politiques économiques et sociales d'austérité de l'UE, elles continuent néanmoins de

\footnotetext{
4. Une conclusion similaire s'applique à la notion de " populisme " qui a, elle aussi, occupé une large place dans l'espace médiatique à l'occasion du scrutin de mai 2014. En termes systémiques, la transition démocratique en Europe orientale et centrale a historiquement produit une forme de populisme " mainstream » qu'incarnent par exemple des partis tels que le FIDESZ de Viktor Orban en Hongrie, Droit et Justice (PiS) de Jaroslaw Kaczynski en Pologne ou le SMER de Robert Fico en Slovaquie. Plus récemment, de nouveaux acteurs sont venus saturer un peu plus l'espace du populisme "centriste » à l'Est de l'Europe: ainsi le mouvement de Janusz Palikot en Pologne, le parti Affaires Publiques (VV) en République Tchèque ou, en Slovaquie, Liberté et Solidarité (SaS) fondé par l'homme d'affaires Richard Sulik.

5. Cf. Vincenzo Emanuele, Nicola Maggini et Bruno Marino (2014) « Gaining Votes in Europe Against Europe? The Electoral Performance of Anti-EU Parties in the 2014 European Elections, » Papier présenté à la Conférence Générale de l'ECPR, Université de Glasgow, 5 septembre (http://ecpr.eu/Filestore/PaperProposal/98df836dabef-4799-bc8a-3a560ecacd2f.pdf)
} 
témoigner de leur attachement au projet européen, se distinguant par là même de l'euroscepticisme dur.

\section{Focus sur les droites radicales populistes}

Pour les raisons que l'on vient d'évoquer, les partis qui gravitent aujourd'hui dans la grande constellation eurosceptique ne constituent pas un même enjeu, moins encore une même menace, pour les démocraties nationales ou l'Union européenne. Il convient ici de resserrer le focus sur une catégorie spécifique d'acteurs. Situés à la droite de l'échiquier politique, ceux-ci sont caractérisés avant tout par la radicalité de leur projet politique, au-delà de l'opposition à l'Europe ou de leurs diatribes anti-establishment. Pour partie, en effet, le succès des forces eurosceptiques en 2014 a été porté par les protagonistes de cette « droite radicale populiste ${ }^{6}$, avatars d'une nouvelle extrême droite paneuropéenne, dont le projet se définit en opposition frontale avec les valeurs fondatrices de l'Union' ${ }^{7}$ Cette extrême droite plus "moderne ", dont le Front national français représente l'archétype, s'est développée en Europe occidentale depuis la fin des années 1970. Ses leaders se sont émancipés en apparence du legs encombrant du fascisme ou du national-socialisme, pour mieux intégrer le jeu démocratique régulier, jusquà obtenir dans certains cas le statut de partenaire de coalition pour les conservateurs, libéraux ou chrétiens-démocrates ${ }^{8}$.

En politisant de nouveaux enjeux culturels, ces partis ont progressivement structuré une offre idéologique nationaliste ethnocentriste, se posant en remparts contre les "dangers " représentés par l'immigration, les minorités ou, désormais, l'islam. Leur repli identitaire est assorti d'un chauvinisme du welfare, c'est-à-dire la volonté de réserver aux seuls nationaux l'accès aux ressources et à la richesse nationale. Ici, l'euroscepticisme prend à l'évidence racine dans ce discours nationaliste, mais ne constitue cependant qu'une facette seulement d'un corpus idéologique plus large. Leur modèle de société demeure dans la plupart des cas empreint d'autoritarisme et de conservatisme social, qui positionnent ces partis à l'extrémité droite de l'axe politique sur les questions relatives à la sécurité, aux mœurs et plus généralement aux libertés individuelles. Politiquement, enfin, ces acteurs se posent en porte-parole du "peuple " contre des "élites " jugées "dévoyées " ou "corrompues». Au fil du temps, leur populisme anti-système leur a permis d'agréger de multiples formes de protestation ou de ressentiment à l'encontre des

6. Voir à ce propos le travail de Cas Mudde, Populist Radical Right Parties in Europe, (Cambridge: Cambridge University Press 2007).

7. Cf. Catherine Fieschi, «European institutions: the far-right and illiberal politics in a liberal context, " Parliamentary Affairs 53, (2000): 517-531.

8. Cf. Gilles Ivaldi, 2005, «À propos du succès des droites populistes et extrêmes en Europe: éléments pour une analyse systémique ", Politeia, "Souverainisme, nationalisme, Régionalisme (2) ", nº 7, printemps, pp.293-306. 
décideurs politiques, de "l'establishment » ou, pour reprendre les termes du FN, de la " caste " politique nationale et européenne. Parce qu'ils offrent un nouveau visage et un discours plus euphémisé, prenant soin en outre de ne pas assaillir de front les piliers de la démocratie représentative, ces acteurs radicaux populistes constituent un nouveau défi pour les normes et valeurs libérales prédominantes au sein des nations européennes. Leur culture politique, faite d'arbitraire et de refus du pluralisme, attaque directement les compromis institutionnels, garanties constitutionnelles et autres corps intermédiaires pourtant essentiels à la stabilité des régimes démocratiques.

La présence de cette offre idéologique nationaliste, autoritaire et anti-establishment permet d'identifier les mouvements qui composent aujourd'hui la galaxie des droites radicales populistes. Au total, 25 partis de ce type étaient en lice à l'occasion du scrutin de mai dernier et certains d'entre eux y ont tenu le devant de la scène. À l'instar du Front national en France, il s'agit souvent d'acteurs partisans établis de longue date sur la scène politique européenne, dont les origines remontent aux années 1970 ou 1980, tels le Parti du Peuple danois (DF), le FPÖ autrichien, le Vlaams Belang en Belgique ou la Ligue du Nord en Italie. D'autres acteurs majeurs de la famille radicale populiste évoluent hors des frontières de l'UE: ainsi le Parti du Progrès (FrP) en Norvège ou l'UDC/SVP suisse, qui occupent tous deux des places centrales au sein de leurs systèmes partisans respectifs. Si l'on se tourne vers l'Europe orientale et centrale, la droite radicale populiste s'incarne principalement dans des mouvements tels que le Parti de la Grande Roumanie (PRM), ATAKA en Bulgarie, le parti Ordre et Justice (TT) Lituanien ainsi que les deux petits Partis nationaux slovaque et slovène (SNS). Plus récemment, les performances électorales de la formule idéologique radicale populiste ont attiré de nouveaux acteurs qui ont choisi d'émuler le modèle de mobilisation xénophobe, autoritaire et populiste incarné, notamment, par le FN. C’est le cas du PVV de Geert Wilders aux Pays-Bas, des Vrais Finnois (PS) en Finlande ou de l'UKIP britannique dont les évolutions récentes témoignent d'une radicalisation et d'un certain mimétisme du parti de Marine Le Pen en dépit d'une volonté stratégique de Nigel Farage de continuer de se démarquer politiquement de ce dernier. Enfin, à la périphérie de la droite radicale populiste évoluent des formations plus classiques de l'extrême droite traditionnelle, directement liées à l'héritage doctrinal du fascisme et souvent marquées encore au sceau de l'activisme violent, à l'image du British National Party (BNP), du NPD allemand, des Démocrates Suédois (SD), du Jobbik en Hongrie ou, plus récemment, de l'Aube Dorée (XA) dont les succès électoraux attestent d'une résurgence inquiétante du néo-nazisme en Grèce depuis $2012^{9}$.

9. La formation de Nikolaos Michaloliakos a remporté $7 \%$ des voix et 21 élus lors des législatives de mai 2012, un score confirmé un mois plus tard lors des législatives anticipées de juin avec $6.9 \%$ des suffrages et 18 sièges. 
Nous aurons l'occasion de le souligner, les enjeux qui structurent le vote en faveur de ces partis demeurent très spécifiques et ne recouvrent que partiellement les questions économiques ou sociales qui ont dominé l'agenda européen en mai dernier. À l'inverse, l'absence d'une ou plusieurs composantes du noyau idéologique typique des droites radicales incite à la prudence. Citons ici le cas du M5S italien de Beppe Grillo dont le populisme anti-establishment fait clairement écho au discours anti-élites des droites radicales, mais qui se tient très largement à distance par ailleurs du discours autoritaire ethnocentriste de partis tels que le FPÖ ou le FN. Pour les mêmes raisons, les acteurs radicaux populistes se différencient d'autres formations de la droite eurosceptique tels que le parti des citoyens libres (Svobodni) en République tchèque, Debout la République (DLR) de Nicolas Dupont-Aignan en France ou l'Alliance nationale lettone (TB/LNNK). C'est également le cas pour certains partis single-issue (enjeu unique) dont la seule raison d'être reste l'antagonisme à l'UE, à l'image de la petite formation EUSTOP en Autriche ou du Mouvement populaire contre l'UE au Danemark.

Dans plusieurs autres cas, l'absence d'une dimension xénophobe ou de discours hostiles aux minorités laisse, pour l'heure, des mouvements tels que l'AfD en Allemagne, les nationaux-conservateurs de l'Alliance démocratique (HDSSB) en Croatie ou les eurosceptiques « soft » de Bulgarie sans Censure (BbC) hors du périmètre des droites radicales populistes stricto sensu. Sans préjuger naturellement de possibles convergences idéologiques à l'avenir. À ce titre, on suivra avec intérêt dans les prochains mois la trajectoire du Congrès de la Nouvelle Droite (KNP) polonais du très controversé Janusz Korwin-Mikke ${ }^{10}$. Avec 7,2\% des suffrages aux Européennes, ce dernier semble à même de s'installer dans l'espace politique de la droite anti-establishment en Pologne, une position occupée auparavant par les populistes ultra-conservateurs de la Ligue des Familles polonaises notamment, qui pourrait le rapprocher progressivement des droites radicales européennes.

\section{Une percée de la droite radicale populiste?}

Au soir du 25 mai, le succès des partis de la droite radicale est resté somme toute relatif, quand bien même certains résultats ont pu accréditer l'idée d'une "vague populiste » paneuropéenne. Fait quasiment sans précédent dans l'histoire électorale de l'Europe depuis $1945^{11}$, ces partis ont, il est vrai, viré en tête du scru-

10. Le leader du KNP s'est fait remarquer pour ses propos polémiques pendant la campagne européenne, déclarant qu'Hitler n'aurait pas, selon lui, été informé de l'Holocauste. Korwin-Mikke s'est également distingué par son soutien à Vladimir Poutine dans la crise ukrainienne ou ses commentaires sur les victimes de viol prétendument "consentantes" (Cf. http://blogs.lse.ac.uk/europpblog/2014/06/10/the-congress-of-thenew-right-is-the-latest-anti-establishment-party-to-have-success-in-poland-but-it-may-struggle-to-secure-longterm-support/).

11. À l'exception toutefois du cas helvétique où les populistes de l'UDC/SVP ont remporté toutes les élections fédérales depuis 2003, réunissant $26,6 \%$ des suffrages lors du dernier scrutin d'octobre 2011. 
tin dans trois États membres - en Grande-Bretagne où l'UKIP a réuni 26,8\% des voix, au Danemark où le Parti du peuple a recueilli $26,6 \%$ des suffrages et en France où le Front national a totalisé $25 \%$ des voix. Avec plus de force symbolique encore, les élections de 2014 ont également vu l'entrée au parlement de deux formations néo-nazies, le NPD allemand d'Udo Voigt (1\%, 1 siège) et l'Aube dorée grecque $(9,4 \%, 3 \text { sièges })^{12}$.

Au total, les représentants de la droite radicale populiste obtiennent 79 élus contre 52 en 2009 et 49 en 2004 (voir Figure 2), soit $11 \%$ seulement des 751 sièges du Parlement européen (contre $7 \%$ en 2009 et 2004). Il s'agit là de gains modérés, compte tenu notamment, nous aurons l'occasion de le souligner, de la forte fragmentation de cette famille politique. Plus encore, l'augmentation constatée en mai dernier est due, pour l'essentiel, aux scores réalisés par l'UKIP en Grande-Bretagne et par le Front national en France, qui contribuent à eux seuls pour 32 sièges supplémentaires par rapport au scrutin de $2009^{13}$.

Figure 2. Total des sièges obtenus par les partis de droite radicale populiste au Parlement européen (2004-2014)

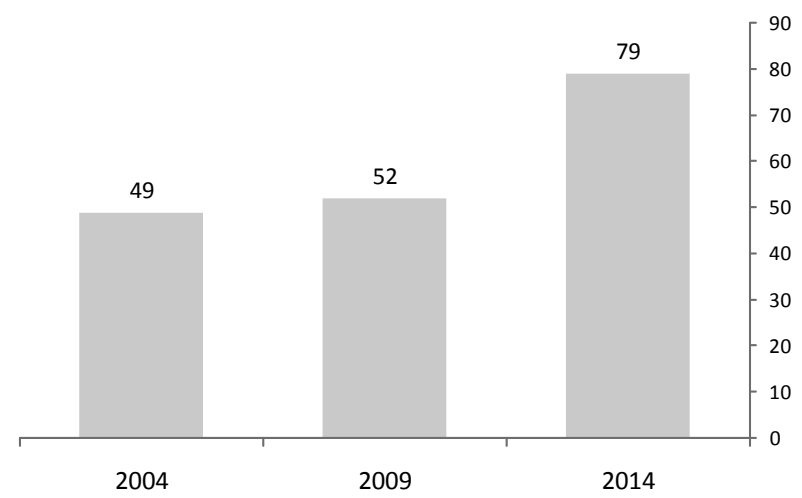

Source: Parlement européen, calculs de l'auteur

Cet apport significatif des populistes britanniques et français vient compenser les pertes subies par d'autres acteurs de la droite radicale populiste. Cette contribution disproportionnelle ne peut masquer la réalité de performances très variables sur l'ensemble du continent européen (voir le détail dans le tableau 1).

12. Là encore, il n'est pas inutile de rappeler que les néo-fascistes italiens du MSI sont présents au Parlement européen depuis 1979, où ils ont longtemps fait alliance avec le Front national de Jean-Marie Le Pen.

13. On notera ici l'impact différentiel lié à la taille des représentations nationales: en dépit d'un score élevé de $26,6 \%$ des voix, les populistes danois du DF n'obtiennent au final que 4 des 13 sièges détenus par le Danemark à Strasbourg (contre, à titre de comparaison, 24 sièges pour le FN français avec un score identique de $25 \%$ des suffrages). 
Tableau 1. Résultats des partis de droite radicale populiste au Parlement européen en 2014

\begin{tabular}{|c|c|c|c|c|c|c|}
\hline Pays & Parti & 2014 & $\begin{array}{l}\text { Sièges } \\
2014\end{array}$ & $\begin{array}{c}\text { Evol. \% } \\
2009- \\
2014\end{array}$ & $\begin{array}{c}\text { Rappel \% } \\
2009\end{array}$ & $\begin{array}{c}\text { Rappel Sièges } \\
2009\end{array}$ \\
\hline Allemagne & NPD & 1,0 & 1 & $+0,6$ & 0,4 & 0 \\
\hline Allemagne & Republikaner & 0,4 & 0 & $-0,9$ & 1,3 & 0 \\
\hline Autriche & FPÖ & 19,7 & 4 & $+7,0$ & 12,7 & 2 \\
\hline Autriche & BZÖ & 0,5 & 0 & $-4,1$ & 4,6 & 0 \\
\hline Belgique & $\begin{array}{c}\text { Vlaams } \\
\text { Belang (VB) }\end{array}$ & 4,1 & 1 & $-5,7$ & 9,9 & 2 \\
\hline Bulgarie & ATAKA & 3,0 & 0 & $-9,0$ & 12,0 & 2 \\
\hline Danemark & $\mathrm{DF}$ & 26,6 & 4 & $+11,3$ & 15,3 & 2 \\
\hline Espagne & FE-JONS & 0,1 & 0 & $+0,1$ & 0,0 & 0 \\
\hline Espagne & $\mathrm{DN}$ & 0,1 & 0 & $+0,1$ & 0,0 & 0 \\
\hline Finlande & $\begin{array}{l}\text { Vrais Finnois } \\
\text { (PS) }\end{array}$ & 12,9 & 2 & $+3,1$ & 9,8 & 1 \\
\hline France & FN & 25,0 & 24 & $+18,7$ & 6,3 & 3 \\
\hline Grèce & $\begin{array}{c}\text { Aube Dorée } \\
\text { (XA) }\end{array}$ & 9,4 & 3 & $+9,4$ & 0,0 & 0 \\
\hline Grèce & La.O.S. & 2,7 & 0 & $-4,4$ & 7,1 & 2 \\
\hline Hongrie & Jobbik & 14,7 & 3 & $-0,1$ & 14,8 & 3 \\
\hline Italie & Lega Nord & 6,2 & 5 & $-4,0$ & 10,2 & 9 \\
\hline Italie & Fratelli-AN & 3,7 & 0 & $+3,7$ & 0,0 & 0 \\
\hline Lituanie & $\begin{array}{c}\text { Ordre et } \\
\text { Justice (TT) }\end{array}$ & 14,3 & 2 & $+2,4$ & 11,9 & 2 \\
\hline Pays-Bas & PVV & 13,3 & 4 & $-3,7$ & 17,0 & 5 \\
\hline Portugal & PNR & 0,5 & 0 & $+0,1$ & 0,4 & 0 \\
\hline Roumaine & PRM & 2,7 & 0 & $-6,0$ & 8,7 & 3 \\
\hline Royaume-Uni & UKIP & 26,8 & 24 & $+10,2$ & 16,6 & 13 \\
\hline Royaume-Uni & $\mathrm{BNP}$ & 1,1 & 0 & $-4,9$ & 6,0 & 2 \\
\hline Slovaquie & SNS & 3,6 & 0 & $-1,9$ & 5,5 & 1 \\
\hline Slovénie & SNS & 4,0 & 0 & $+1,1$ & 2,9 & 0 \\
\hline Suède & $\begin{array}{l}\text { Démocrates } \\
\text { Suédois (Sd) }\end{array}$ & 9,7 & 2 & $+6,4$ & 3,3 & 0 \\
\hline
\end{tabular}


Remarquons tout d'abord que plus de la moitié des États membres restent épargnés par le phénomène et n'ont pas d'élus issus de la droite radicale populiste. En nombre de sièges, ces partis ne progressent que dans huit pays, maintiennent leurs positions dans cinq et reculent au final dans 7 États membres. C'est le cas, notamment, d'ATAKA en Bulgarie, de la Ligue du Nord en Italie, du PRM en Roumanie ou du PVV néerlandais ${ }^{14}$. On notera également dans plusieurs pays des effets de recomposition à l'intérieur même de l'espace de la droite radicale populiste. En Autriche, le maintien du FPÖ de Heinz-Christian Strache se fait pour beaucoup aux dépends d'une BZÖ en voie de marginalisation politique. Dans le cas britannique, l'UKIP semble avoir très largement vampirisé la base électorale du BNP, privant du même coup ce dernier de son siège au Parlement européen. En Belgique, le Vlaams Belang continue de voir une partie de son électorat glisser vers l'Alliance néo-flamande (N-VA), nouvel acteur central de la revendication séparatiste en Flandre, une migration à l'œuvre depuis les législatives de 2010. En Grèce, enfin, l'Aube Dorée s'impose dans un espace radical essentiellement occupé jusque-là par le Rassemblement populaire orthodoxe (La.O.S) de Georgios Karatzaferis avant que ce dernier ne paie le prix électoral de son soutien éphémère au gouvernement intérimaire de grande coalition de Lucas Papademos fin 2011.

\section{Populisme et crise économique}

Cette absence d'une poussée uniforme des forces de la droite radicale populiste à l'échelle du continent rappelle l'importance des cycles et enjeux nationaux dans un scrutin européen traditionnellement perçu comme une élection de «secondordre ». Dans un certain nombre de cas, en effet, les paramètres de l'élection de 2014 répondent aux critères du modèle popularisé par Reif et Schmitt au début des années 1980 : primauté des enjeux nationaux, élection intermédiaire, faible participation, sanction des partis de gouvernement, vote protestataire et essor des forces périphériques ${ }^{15}$. En mai dernier, les systèmes de partis nationaux se sont à nouveau dilatés à l'occasion d'un scrutin européen marqué par la fragmentation du jeu politique, liée pour partie à l'éparpillement des votes sur les partis protestataires et aux performances à la baisse des grands partis de gouvernement ${ }^{16}$. En

14. En dépit de sondages prometteurs jusqu’à la veille du scrutin, le mouvement de Geert Wilders termine in fine en quatrième position avec $13,3 \%$ des voix. Outre une concurrence directe du Parti socialiste (SP) sur le terrain europhobe, le PVV tombe victime de nouveaux propos controverses concernant les marocains en mars 2014. Sans doute la perspective d'une alliance avec le FN a-t-elle également renforcé le sentiment d'un parti en voie de radicalisation idéologique. Plus profondément, la formation de Geert Wilders paie le prix de son soutien au cabinet libéral-chrétien-démocrate de Mark Rutte à partir de 2010, avant d'être responsable de sa chute en avril 2012.

15. Cf. Karlheinz Reif, and Hermann Schmitt, 1980, « Nine second-order national elections - a conceptual framework for the analysis of European election results, "European Journal of Political Research, 8(1): 3-44

16. Dans des pays de culture majoritaire tels que la France ou la Grande-Bretagne, l'usage de la proportionnelle pour le scrutin européen accroît encore l'effet d'opportunité pour les mouvements périphériques et contribue 
2009, la "sanction" des gouvernements en place avait touché 10 des 27 Etats membres; cette année, elle a concerné une douzaine de pays, singulièrement dans la partie occidentale de l'Union ${ }^{17}$. En France, en Autriche, en Grèce, au Danemark ou au Royaume-Uni, les performances des formations radicales populistes se sont inscrites dans ce mouvement national de protestation contre l'exécutif.

Peut-être plus que de par le passé, toutefois, les succès enregistrés par la droite radicale populiste à l'occasion du scrutin de 2014 ont témoigné de l'européanisation croissante du débat politique. On sait depuis plusieurs années que la dimension européenne s'est progressivement alignée sur un ensemble plus complexe d'enjeux relatifs au rapport qu'entretiennent acteurs politiques et citoyens à l'ouverture internationale et plus généralement au processus de "dénationalisation »: la globalisation économique, la création d'un État supranational avec l'UE ou l'immigration constituent notamment de nouvelles questions interdépendantes qui structurent la compétition partisane à la fois sur la scène européenne et dans l'espace national ${ }^{18}$.

Ces enjeux occupent une place centrale dans l'offre programmatique des principaux acteurs de la droite radicale populiste européenne. À la faveur de la crise économique et financière de 2008 , ces derniers ont stratégiquement accentué leur discours anti-globalisation, nationaliste et protectionniste, adoptant pour certains d'entre eux un agenda de redistribution et de préservation des acquis sociaux contre la « menace » immigrée. Des partis tels que le PVV néerlandais, le FPÖ ou le FN français prétendent aujourd'hui être les meilleurs gardiens de l'État providence ou du pouvoir d'achat des plus modestes, critiquant la conversion des partis de gouvernement à l'austérité imposée par l'UE ou le FMI, et rejoignant les positions plus traditionnellement welfaristes des populistes scandinaves, Parti du peuple danois, Parti du Progrès en Norvège ou Démocrates suédois. En France, le Front national de Marine Le Pen a endossé les habits du social-populisme, empruntant à la gauche nombre de ses positions sur les bas salaires, les retraites ou le rôle de l'État en 2012. Sous la houlette de Heinz Christian Strache, le FPÖ autrichien a effectué depuis 2005 un virage comparable à gauche, combinant étatisme et justice sociale, jusqu'à tenter de se présenter comme le nouveau parti de la classe ouvrière. Jusqu'au très néo-libéral Geert Wilders qui s'efforce depuis

\footnotetext{
à cette fragmentation. En Allemagne, le NPD a également profité de l'abaissement du seuil de représentation pour s'arroger un siège malgré un maigre $1 \%$ des suffrages.

17. Ce vote n'a pas favorisé systématiquement pour autant les formations extrêmes: en témoignent les cas du Portugal, de la Bulgarie, de la Slovénie ou de la Suède, où la sanction des gouvernements s'est traduite pour l'essentiel par un soutien aux principaux partis d'opposition.

18. Voir à ce sujet: Hanspeter Kriesi, Edgar Grande, Romain Lachat, Martin Dolezal, Simon Bornschier and Timotheos Frey, eds, West European Politics in the Age of Globalization (Cambridge: Cambridge University Press, 2008).
} 
2010 de donner à son parti un ton plus social pour tenter d'élargir ses soutiens électoraux.

Dans de nombreux cas, les élections européennes de 2014 ont confirmé le renforcement d'une base ouvrière pour les partis de la droite radicale populiste, un phénomène de prolétarisation à l'œuvre depuis une vingtaine d'années au plan national ${ }^{19}$. La pénétration de ces partis au sein de l'électorat populaire est particulièrement visible en France ou en Autriche, où le FN et le FPÖ se sont durablement installés dans d'anciens bastions de la gauche. Aux Pays-Bas, le PVV obtient ses meilleurs scores dans les grandes périphéries urbaines, auprès d'une classe moyenne inquiète pour son statut. De la même manière, les travaux récents sur le phénomène UKIP en Grande-Bretagne montrent comment un parti issu originellement de la droite conservatrice est parvenu en quelques années à s'imposer comme principal challenger du Labour sur les terres ouvrières, au point de constituer une menace sérieuse pour l'opposition travailliste aux prochaines législatives de $2015^{20}$. En France, les listes du Front national auraient, selon les enquêtes, recueilli pas moins de $43 \%$ des voix chez les ouvriers et $38 \%$ auprès des employés en mai dernier ${ }^{21}$. En Autriche, la formation de Heinz-Christian Strache aurait séduit quant à elle près de la moitié ( $46 \%)$ de l'électorat ouvrier, loin devant les sociaux-démocrates du SPÖ $(24 \%)^{22}$.

À ce titre, nombre d'observateurs ont voulu voir dans la prétendue vague populiste européenne la simple manifestation de la crise économique et sociale, réminiscence de la montée en puissance du fascisme dans les années 1930. Les données d'opinion montrent en effet que le contexte économique et financier a fortement pesé sur le scrutin européen dans la plupart des États membres. La situation économique nationale, le chômage et l'état des finances publiques arrivent ainsi en tête des préoccupations sur l'ensemble de l'UE avec respectivement 39,34 et $25 \%$ des réponses, devant l'immigration avec $21 \%$. Près des deux tiers (63\%) des citoyens européens jugent par ailleurs que la situation économique de leur pays est "mauvaise ", un pessimisme qui culmine à plus de $95 \%$ au sud de l'Europe, en Espagne, en Grèce, au Portugal ou en Italie ${ }^{23}$.

S'il se nourrit assurément de la crise, le succès des partis de la droite radicale populiste ne se résume cependant pas exclusivement à la situation de l'économie. Il n'existe pas en tout état de cause de corrélation nette entre les performances

19. Cf. Jens Rydgren (ed.) Class Politics and the Radical Right Right (Abingdon: Routledge, 2013).

20. Cf. Robert Ford et Matthew Goodwin, Revolt on the Right: Explaining Support for the Radical Right in Britain, (Routledge, 2014).

21. Sondage veille du vote - Ipsos/STERIA pour France Télévisions, Radio France, Le Point, LCP Public Senat, France 24 .

22. Sondage SORA, Wahltagsbefragung und Wählerstromanalyse Europawahl 2014 (http://www.sora.at/the$\mathrm{men} /$ wahlverhalten/wahlanalysen/eu-wahl14.html).

23. Eurobaromètre standard $n^{\circ} 81$

L'Europe en formation no373 Automne 2014 - Autumn 2014 
électorales de ces acteurs et les niveaux de chômage ou de croissance dans les divers États membres. Certains des succès les plus remarquables des partis radicaux populistes concernent des nations relativement épargnées par la crise, à l'image de l'Autriche, du Danemark, des Pays-Bas, de la Suède ou du Royaume-Uni, où les opinions publiques se montrent d'ailleurs sensiblement plus optimistes quant à la conjoncture économique: au Danemark, pas moins de $82 \%$ des citoyens interrogés jugent ainsi la situation de leur pays plutôt satisfaisante; ils sont également $81 \%$ en Suède, $60 \%$ aux Pays-Bas ou encore $56 \%$ en Autriche ${ }^{24}$. On pourrait ajouter ici les exemples norvégien et suisse, où les partis populistes se développent dans des contextes similaires de prospérité économique - avec des taux de chômage autour de $3 \%$ de la population active, qui plus est hors du cadre de l'Union européenne.

Plus qu'un effet direct de la récession, la structuration des enjeux économiques montre une démarcation nette entre le noyau des pays riches de l'Union et sa périphérie plus pauvre, notamment au sud et à l'est. En termes régionaux, le phénomène de droite radicale populiste se concentre majoritairement en 2014 sur la partie occidentale du continent: si l'on excepte en effet le succès relatif du Jobbik en Hongrie avec $14,7 \%$ des voix ${ }^{25}$, l'Europe centrale et orientale a vu à l'occasion du scrutin européen un déclin des principaux partis de la famille populiste, notamment en Bulgarie, en Roumanie ou en Slovaquie. Politiquement, la structure de compétition à l'est de l'Europe demeure caractérisée par une plus forte volatilité et une plus grande fluidité des systèmes partisans, systèmes dans lesquels les mouvements de la droite radicale populiste affrontent en outre la concurrence directe d'une pluralité d'acteurs, pour certains plus " mainstream ", capables d'articuler une offre populiste, anti-establishment et eurosceptique. Le déclin des droites radicales en Europe orientale nous rappelle également que, dans des pays tels que la Pologne, la Slovaquie ou les États baltes, les fonds structurels de l'UE ont agi comme de formidables moteurs de développement économique.

Il est intéressant de constater qu'en mai dernier, le poids électoral des mouvements de droite radicale populiste a été sensiblement plus élevé dans les États contributeurs au budget de l'UE où ces partis ont obtenu $17 \%$ des voix en moyenne, contre à peine $5 \%$ dans les pays bénéficiaires. À l'exception notable de la Grèce, nous n’avons pas assisté dans les pays bénéficiaires de plans d'aide de l'UE à l'émergence ou à la poussée de ce type de mouvements ${ }^{26}$. À l'inverse, la

24. En comparaison, le niveau de satisfaction avec la situation économique nationale chute à $8 \%$ en France (Eurobaromètre standard $n^{\circ} 81$ ).

25. Aux législatives d'avril 2014, le parti de Gabor Vona avait réuni 20,6 \% des suffrages, se plaçant en troisième position derrière les conservateurs du FIDESZ et la coalition des partis de centre-gauche (Unité) emmenée par Attila Mesterhazy.

26. D’autres types d'acteurs y ont incarné l'expression politique du ressentiment économique: ainsi la Coalition de la Gauche radicale (SYRIZA) d'Alexis Tsipras en Grèce, la Gauche Plurielle (IP) et Podemos en Espagne, 
rébellion des droites radicales a été particulièrement marquée dans certains pays riches de l'Union, situés notamment à l'extérieur de la zone Euro, à l'image du Royaume-Uni ou du Danemark. Cette distribution différentielle fait directement écho à l'existence d'un "populisme des riches " que l'on retrouve plus généralement au travers de la présence forte des droites radicales populistes dans des certaines des nations les plus affluentes d'Europe occidentale - Autriche, Suisse, Norvège ou Danemark - ainsi que dans certaines régions riches telles la Flandre ou l'Italie du Nord.

Elle est également révélatrice des positions prises par ces partis dans la crise financière et $\mathrm{du}$ " chauvinisme de la dette " dont ils se sont faits les porte-voix. Très vite, au plus fort de la tourmente financière de 2008, des partis tels que les Vrais Finnois, le PVV, le FN ou le FPÖ ont dénoncé le "prix à payer " pour sauver l'Euro et refuser les plans d'aides de l'Union européenne. Timo Soini, leader des Vrais Finnois, a, pour partie, bâti le succès de sa formation aux législatives de 2011 sur cette contestation directe du sauvetage des pays d'Europe du Sud, dénonçant le "racket " imposé aux contribuables finlandais. En France, Marine Le Pen et le Front national ont mené une campagne virulente contre ces mêmes plans de renflouement sur le thème «Grèce et Euro: les Français n'en peuvent plus de payer! ». En mai 2012, Geert Wilders s'est opposé avec véhémence au vote du Mécanisme européen de stabilité par le gouvernement néerlandais. Côté autrichien, le FPÖ de Heinz-Christian Strache s'est également élevé contre ce qu'il considère être une "expropriation de masse des Autrichiens ", comparant ce mécanisme au Ermächtigungsgesetz allemand des années 1930, texte qui avait permis l'accession de Hitler au pouvoir. En Italie, sous la nouvelle direction de Matteo Salvini, la Lega Nord a pris, fin 2013, un net tournant anti-européen et rejoint notamment le Front national sur la sortie de l'Euro (Basta Euro, Sortir de l'Euro, vite!), une convergence programmatique qui a présidé à la décision de la Ligue en janvier 2014 de rejoindre officiellement le FN et ses partenaires de l'Alliance européenne pour la Liberté (AEL).

L'absence de corrélation directe entre crise économique et performances des droites radicales populistes nous ramène enfin à la nature même de la spécificité de leur mobilisation sur des enjeux principalement culturels, en particulier l'immigration. Le nouvel agenda économique de ces mouvements, dont on souligne souvent la proximité d'avec la plateforme protectionniste et anti-globalisation de certains partis de gauche antilibérale, ne peut dissimuler le rôle central du nationalisme xénophobe et du chauvinisme de l'État providence ${ }^{27}$. En mai der-

le Sinn Fein en Irlande, l'Action des Citoyens mécontents (ANO) en République tchèque ou le M5S de Beppe Grillo en Italie.

27. Cf. Zhirkov, Kirill (2014) « Nativist but not alienated. A comparative perspective on the radical right vote in Western Europe, " Party Politics, 20(2): 286-296. 
nier, l'immigration, le contrôle des frontières et la défense du welfare ont été au cœur des campagnes menées par les formations de la droite radicale populiste. En Grande-Bretagne, l'UKIP a séduit sur le rejet du fédéralisme mais a surtout centré son propos sur l'immigration, la criminalité et l'impact négatif à ses yeux de la libre circulation sur le système éducatif, le logement ou la santé. De la même façon, les populistes du Parti du peuple danois conduits par Morten Messerschmidt ont bâti leur succès sur la baisse des droits sociaux pour les immigrés issus de l'UE, le contrôle des frontières et la lutte contre le dumping social. En France, Marine Le Pen et le Front national ont également placé l'immigration au cœur du débat européen, dénonçant notamment les effets "délétères » de la directive détachement et demandant une remise à plat des accords de Schengen ${ }^{28}$. Des thèmes de campagne similaires à ceux mis avant par le FPÖ emmené par Harald Vilimsky en Autriche: immigration, criminalité, contrôle des frontières et sortie de Schengen, en particulier ${ }^{29}$. En Italie, la Lega a fait de la lutte contre l'immigration illégale un point dominant de son programme sur le thème "Arrêtons l'invasion ", proposant notamment de restaurer le crime de "clandestinité " pour les immigrés. En Hongrie, enfin, Jobbik a mené une campagne en faveur de "l'Europe des nations » et de la défense des intérêts et droits sociaux des salariés hongrois.

\section{Un péril pour l'Europe?}

Annoncée mi-novembre 2013, la création d'une fédération de partis autour de l'Alliance européenne pour la Liberté (AEL) représente une nouvelle tentative par le Front national de Marine Le Pen d'affirmer son leadership européen sur l'espace des droites radicales. Rappelons que le FN français a été au centre de toutes les entreprises de rassemblement au cours des trente dernières années, depuis le groupe des Droites européennes entre 1984 et 1994 jusqu’à l'Alliance européenne des Mouvements nationaux en 2009 passant par le réseau EURONAT au milieu des années 1990 ou le très éphémère groupe Identité, Tradition, Souveraineté (ITS) en 2007. L'enthousiasme récent du PVV néerlandais, du FPÖ autrichien ou de la Lega Nord illustre aussi le turn over générationnel à la tête de ces divers partis: Marine Le Pen a succédé à son père en janvier 2011; HeinzChristian Strache occupe désormais l'espace du populisme autrichien incarné par Jörg Haider jusqu'à sa mort en 2008; en Italie, le charismatique père fondateur de

28. L'immigration est d'ailleurs arrivée en tête des préoccupations de l'électorat hexagonal le 25 mai, citée comme l'enjeu le plus important par $31 \%$ des interviewés, devant le pouvoir d'achat $(30 \%)$ et la crise dans la zone Euro (27 \%) (Sondage veille du vote - Ipsos/STERIA pour France Télévisions, Radio France, Le Point, LCP Public Senat, France 24).

29. L'électorat autrichien dans son ensemble a placé l'immigration au cœur du vote européen, en première position dans la liste des questions les plus importantes, devant le chômage et la lutte contre la criminalité (SORA, Wahltagsbefragung und Wählerstromanalyse Europawahl 2014). 
la Lega, Umberto Bossi, a finalement passé la main et ouvert la voie à Matteo Salvini en 2012. Ces dirigeants, plus jeunes, incarnent une nouvelle génération de leaders plus pragmatiques et désireux d'asseoir plus fortement encore l'influence politique de leurs formations respectives.

L'échec de Marine Le Pen à former un groupe au Parlement européen a toutefois témoigné du succès en trompe l'œil de ces mouvements. Le FN a souffert de la concentration en sièges sur un petit nombre de partis ${ }^{30}$ et des mauvais résultats enregistrés par plusieurs de ses partenaires potentiels, en particulier en Slovaquie où le Parti national (SNS) avait annoncé son intention de se rapprocher de l'AEL avant d'échouer aux portes du Parlement de Strasbourg. Il demeure naturellement possible pour la présidente du Front national de trouver ultérieurement des soutiens minoritaires auprès de députés sécessionnistes en rupture de ban avec leurs partis d'origine. Un renfort inattendu pourrait ainsi venir d'un nouveau " maverick» issu des rangs de l'UKIP britannique comme cela avait été le cas en 2007. Les yeux de Marine Le Pen sont également tournés vers les Démocrates suédois, l'Alliance Nationale lettone (1 siège) ou, plus probablement, le KPN polonais dont 3 des 4 élus siègent encore pour l'heure parmi les non-inscrits.

Tableau 2. Réseaux transnationaux des droites radicales populistes depuis 1984

\begin{tabular}{|c|c|c|}
\hline Période & Nom & Partis membres \\
\hline $1984-1994$ & Groupe des droites européennes & $\begin{array}{l}\text { FN, MSI, Vlaams Blok, } \\
\text { Republikaner, EPEN }\end{array}$ \\
\hline $1997-$ & EURONAT & $\begin{array}{l}\text { Réseau transeuropéen de } 21 \text { partis, } \\
\text { dont FN, Vlaams Blok, DVU, MSI- } \\
\text { FT, MIEP, PRM, BNP ou SD }\end{array}$ \\
\hline 2007 & Identité, Tradition, Souveraineté (ITS) & $\begin{array}{c}\text { FN, Vlaams Belang, FPÖ, MSI-FT, } \\
\text { ATAKA, PRM }{ }^{(1)}\end{array}$ \\
\hline $2009-2013$ & $\begin{array}{l}\text { Alliance européenne des Mouvements } \\
\text { nationaux }^{(2)}(\mathrm{AEMN})\end{array}$ & $\begin{array}{c}\text { FN, FPÖ, MSI-FT, BNP, FN } \\
\text { wallon, PNR, ND, MIEP-Jobbik }\end{array}$ \\
\hline $2010-2014$ & $\begin{array}{c}\text { Alliance européenne pour la Liberté } \\
\text { (AEL) }\end{array}$ & $\begin{array}{c}\text { FN, FPÖ, Vlaams Belang, PVV, } \\
\text { Lega Nord }\end{array}$ \\
\hline
\end{tabular}

(1) Auxquels s'ajoutait un député européen indépendant britannique, ancien membre de l'UKIP.

(2) Avant le départ des représentants du FN français en octobre 2013.

Cette incapacité de fédérer les droites radicales populistes reste cependant emblématique de la fragmentation endémique dont souffre cette famille politique depuis 1979. L'histoire de ces mouvements au sein du Parlement européen a été

30. Rappelons qu'il faut au minimum 25 députés répartis dans 7 États membres pour former un groupe au Parlement de Strasbourg. 
marquée par la forte volatilité des alliances qu'ils ont été en mesure de forger au fil du temps, entre options idéologiques contradictoires, irrédentismes nationaux ou simplement lutte pour le leadership ${ }^{31}$. Les désaccords entre partis de la droite radicale populiste montrent les limites d'alliances essentiellement tactiques, qui se heurtent dans de nombreux cas à la prédominance d'intérêts partisans. À plusieurs reprises de par le passé, certains de ces partis ont stratégiquement évité un rapprochement avec d'autres membres de la famille radicale au motif de leur « extrémisme ", dans le but souvent d'asseoir leur propre légitimité et de se dédouaner d'accusations similaires. En témoigne par exemple le refus récent de Nigel Farage d'associer l'UKIP à l'AEL de Marine Le Pen. N'oublions pas non plus que le néerlandais Geert Wilders a longtemps gardé ses distances avec un Front national qu'il considérait, à l'époque, comme un mouvement d'extrême droite. Une attitude partagée par les populistes danois du DF dont l'ancienne chef de file, Pia Kjærsgaard, a systématiquement refusé de coopérer avec des formations telles que le FPÖ autrichien ou le FN.

De réelles divergences idéologiques séparent également ces partis. Si les formations radicales populistes partagent volontiers une aversion commune pour les «bureaucrates » de l'Union, elles n'entretiennent pas toutes le même rapport aux institutions européennes et differrent dans leurs objectifs politiques (voir le tableau 3). Nombre de ces partis se retrouvent sur l'hostilité à l'Euro et aux politiques d'austérité qui y sont à leurs yeux associées, mais leurs positions varient plus fortement en revanche sur l'opportunité d'une sortie de l'Union européenne telle que la suggèrent notamment le FN français, le PVV néerlandais ou l'UKIP britannique. La campagne de 2014 a révélé les hésitations en la matière du FPÖ autrichien en dépit de son alliance objective avec le parti de Marine Le Pen. S'agissant encore des grands enjeux stratégiques et géopolitiques, une ligne de fracture traverse la famille radicale populiste, entre formations atlantistes (PVV ou UKIP notamment) et partis marqués par l'anti-américanisme tels le FN français ou la Lega italienne.

Plus fondamentalement enfin, la fragmentation des droites radicales populistes traduit les divergences qui existent en termes de trajectoires politiques. Depuis la fin des années 1970, l'évolution de ces mouvements a été caractérisée par une oscillation entre " radicalité » et " normalisation », entre la volonté d'intégrer le mainstream et la nécessité électorale qui leur impose souvent de préserver leur potentiel protestataire d'opposition intégrale au "système ». De nombreuses formations de la droite radicale populiste ont ainsi fait l'expérience, souvent malheureuse, de la participation directe au pouvoir depuis une quinzaine d'années:

31. Cf. Gilles Ivaldi, "The Populist Radical Right in European Elections: 1979-2009” in Backes (Uwe), Moreau (Patrick) (eds.), The Extreme Right in Europe: Current Trends and Perspectives, Vandenhoeck \& Ruprecht, Hannah Arendt Institute for Research on Totalitarianism, (2012), pp.15-34. 
FPÖ autrichien, Lega Nord en Italie, Parti du Progrès en Norvège, La.O.S. en Grèce, la défunte Liste Pim Fortuyn aux Pays-Bas ou le petit Parti national slovaque entre 2006 et 2010. Ailleurs en Europe, des partis tels que le DF danois ou le PVV de Geert Wilders aux Pays-Bas ont choisi de demeurer en dehors du gouvernement mais d'apporter leur soutien parlementaire aux coalitions en place. D'autres, enfin, restent pour l'heure reclus aux marges du système politique. C'est le cas notamment pour les groupes les plus clairement identifiables à l'extrême droite traditionnelle, tels le NPD allemand, le Jobbik hongrois, le BNP britannique ou l'Aube Dorée en Grèce. Ainsi, également, un ensemble d'acteurs qui demeurent encore entachés de leurs origines extrémistes en dépit de leur volonté affichée de "dédiabolisation": on songe naturellement ici au FN français, mais aussi au Vlaams Belang flamand, à ATAKA en Bulgarie, au PRM roumain ou, plus récemment, aux Démocrates suédois $(\mathrm{Sd})$.

Tableau 3. Aperçu des positions des principaux partis radicaux populistes sur l'Europe

\begin{tabular}{|c|c|c|c|}
\hline Pays & Parti & Sortie zone Euro ${ }^{(I)}$ & Sortie $U E^{(l)}$ \\
\hline Autriche & FPÖ & $\mathrm{X}$ & $\mathrm{O}$ \\
\hline Belgique & Vlaams Belang & $\mathrm{X}$ & $\mathrm{X}$ \\
\hline Bulgarie & ATAKA & Non membre & $\mathrm{O}$ \\
\hline Danemark & $\mathrm{DF}$ & Non membre & $\mathrm{O}$ \\
\hline Finlande & PS & $\mathrm{O}$ & $\mathrm{O}$ \\
\hline France & $\mathrm{FN}$ & $\mathrm{X}$ & $\mathrm{X}$ \\
\hline Hongrie & Jobbik & Non membre & $\mathrm{O}$ \\
\hline Italie & Lega Nord & $\mathrm{X}$ & $\mathrm{O}$ \\
\hline Pays-Bas & PVV & $\mathrm{X}$ & $\mathrm{X}$ \\
\hline Roumanie & PRM & Non membre & $\mathrm{O}$ \\
\hline $\begin{array}{l}\text { Royaume- } \\
\text { Uni }\end{array}$ & UKIP & Non membre & $\mathrm{X}$ \\
\hline Suède & SD & Non membre & $\mathrm{X}$ \\
\hline
\end{tabular}

(1) Cas où les partis envisagent une sortie de la zone Euro et/ou de l'Union européenne, assortie ou non de conditions spécifiques. 
Tableau 4. Distribution transnationale des partis radicaux populistes européens

\begin{tabular}{cccc} 
Membres du Parlement européen (2014) & \\
Non Inscrits & $\begin{array}{c}\text { Alliance européenne } \\
\text { pour la liberté (AEL) }\end{array}$ & $\begin{array}{c}\text { Groupe Europe } \\
\text { libertés démocratie } \\
\text { directe (ELDD) }\end{array}$ & $\begin{array}{c}\text { Groupe des } \\
\text { Conservateurs et } \\
\text { réformistes européens } \\
\text { (CRE) }\end{array}$ \\
$\begin{array}{c}\text { Jobbik, NPD, } \\
\text { Aube Dorée (XA) }\end{array}$ & $\begin{array}{c}\text { FN, PVV, FPÖ, } \\
\text { VB, Lega Nord }\end{array}$ & $\begin{array}{c}\text { UKIP, Ordre et } \\
\text { Justice (TT), } \\
\text { Démocrates } \\
\text { Suédois (Sd) }\end{array}$ & Vrais Finnois (PS), DF \\
ATAKA, PRM, & Partis non représentés au Parlement européen & \\
BNP, FE-JONS, & BZÖ, FN Wallon, PP, & & \\
DN, & SNS slovaque, SNS & La.O.S & \\
Fratelli-AN, & slovène, AN maltaise & \\
PNR & & & \\
\hline
\end{tabular}

Une telle diversité de trajectoires est illustrée par la distribution des acteurs radicaux populistes au sein des fédérations transnationales (Cf. Tableau 4). Plusieurs de ces partis poursuivent aujourd'hui leur entreprise d'intégration systémique $^{32}$. Depuis 2010, la consolidation par Marine Le Pen de l'Alliance européenne pour la Liberté traduit cette volonté de la présidente du FN de rechercher des partenariats avec des partis moins clairement marqués à l'extrême droite traditionnelle. Bien qu'encore inaboutie, cette tentative de "recentrage " a conduit notamment à l'abandon de la très controversée AEMN co-présidée par Bruno Gollnisch aux côtés des extrémistes du BNP britannique ou du Jobbik hongrois, Marine Le Pen ayant décrété ces derniers infréquentables pour un FN en voie de "dédiabolisation " fin 2013. Ce processus de "mainstreaming" se traduit également par le mouvement centripète de partis tels que le DF danois ou les Vrais Finnois de Timo Soini, qui quittent l'ELDD pour rejoindre le groupe plus établi des Conservateurs et Réformistes européens (CRE) aux côtés de partis eurosceptiques d'envergure gouvernementale. De manière similaire, les Démocrates suédois $(\mathrm{Sd})$ ont choisi au final de se démarquer de l'Alliance européenne pour la Liberté de Marine Le Pen au bénéfice du groupe plus « respectable » de l'ELDD dirigé par l'UKIP britannique de Nigel Farage, dans l'objectif annoncé par Jim-

32. On notera ici que certaines des formations les plus ouvertement extrémistes ont initié depuis plusieurs années un même travail d'euphémisation de leur discours et de recherche d'une plus grande " respectabilité " politique. C'est le cas notamment du BNP britannique qui, sous l'impulsion de Nick Griffin, a tenté sans grand succès depuis 1999 de se détacher de son patrimoine néo-fasciste et de l'activisme violent. Les campagnes électorales de 2014 - législatives et européennes - en Hongrie ont également laissé entrevoir une même volonté de la part du Jobbik de modérer son image, dans l'objectif de concurrencer directement le FIDESZ de Viktor Orban au sein de l'espace des droites. 
mie Åkesson de préparer au mieux les législatives de la mi-septembre. À l'inverse, on l'a souligné, des partis tels que le PVV néerlandais, la Lega Nord en Italie ou le SNS slovaque, en prenant la décision de se rapprocher de l'AEL de Marine Le Pen, semblent faire preuve d'une certaine radicalisation idéologique, notamment sur leurs positions vis-à-vis de l'Union européenne et/ou de l'Euro.

\section{Perspectives}

En dépit de certains succès notables, beaucoup des partis de la droite radicale populiste se trouvent aujourd'hui marginalisés au sein d'un Parlement européen qui demeure encore largement dominé par les forces pro-UE. Leur isolement dans l'espace européen renvoie à l'ostracisme dont font preuve à leur encontre les grands partis nationaux : parmi les principaux acteurs de la famille radicale populiste, nombreux sont ceux privés pour l'heure d'alliances au sein de leurs systèmes politiques respectifs - ainsi le FN français, le PVV néerlandais, le FPÖ autrichien, l'UKIP en Grande-Bretagne ou les Démocrates suédois. En panne de dynamique collective, sans perspective de coalition ni véritable capacité de blocage des institutions de l'Union, les partis radicaux populistes devraient néanmoins trouver au sein du parlement de Strasbourg une base de repli stratégique et un ensemble non négligeable de ressources médiatiques, politiques et financières dans les mois et les années à venir.

Ces formations conservent en outre un important pouvoir de nuisance et d'influence. Leur présence au sein du Parlement leur offrira incontestablement une tribune pour continuer leur travail minutieux de délégitimation des institutions, des élites et des politiques de l'Union. Le populisme anti-establishment demeure un moteur fort de mobilisation pour les droites radicales. Dans des pays comme l'Autriche, les Pays-Bas ou la Finlande, pour ne citer ici que quelques exemples, l'existence même d'une culture de coopération entre les principaux partis de gouvernement a nourri le ressentiment à l'encontre des élites partisanes. La formation parfois de "grandes coalitions " y a alimenté l'imagerie populiste d'une " caste " politique, où gauche et droite se confondraient dans un même ensemble indifférencié. Il n'est sans doute pas inutile de rappeler ici que ce modèle de démocratie consensuelle de recherche de compromis prévaut historiquement au sein des institutions européennes. Il constituera de ce fait à l'avenir une cible de choix pour tous les démagogues populistes européens.

Au plan national, les élections européennes nous ont livré en outre des informations sur l'état du rapport de forces politique au sein de chaque État membre. La poussée enregistrée par les Démocrates suédois aux législatives du 14 septembre dernier, en troisième position avec $12,9 \%$ des voix, illustre clairement le lien qui existe plus généralement entre performances européennes et scores natio- 
naux $^{33}$. Les observateurs se tournent déjà vers le Royaume-Uni, le Danemark ou la Finlande pour les prochaines élections générales de 2015, où seront évidemment scrutés les résultats de l'UKIP, du DF et des Vrais Finnois. En France, le poids du FN pèsera immanquablement sur les équilibres politiques et électoraux jusqu'en 2017. Côté autrichien, la bonne tenue européenne du FPÖ maintient la pression sur la grande coalition formée par les conservateurs de l'ÖVP et les sociaux-démocrates du SPÖ.

Au-delà, l'impact des droites radicales populistes est sensible dans la structuration même des agendas nationaux, du débat et des politiques publiques, notamment dans les secteurs clés de l'immigration, de l'asile ou de l'intégration. L'installation durable de cette famille politique dans l'espace européen n'est pas totalement étrangère au durcissement des politiques d'immigration ou d'asile en France, en Italie, en Autriche ou aux Pays-Bas, de même qu'elle a très largement contribué à politiser les questions relatives à l'Islam dans ces mêmes pays depuis plusieurs années. Au plan européen, cette présence est perceptible, de manière plus détournée sans doute, dans les positions prises par les chefs d'État et de gouvernement au Conseil européen, c'est-à-dire au cœur même du pouvoir politique de l'UE. Peut-être plus que par leur présence symbolique au sein de l'hémicycle à Strasbourg ou à Bruxelles, c'est au travers de cette influence indirecte sur les grandes orientations de l'Union européenne que le poids des forces radicales populistes pourrait se faire le plus fortement sentir à l'avenir.

\section{Résumé}

Les élections européennes de mai 2014 ont été dominées par la poussée des forces eurosceptiques. La nature de cette mobilisation demeure toutefois profondément hétérogène, entre euroscepticisme « soft » mainstream et formes plus radicales d'opposition à la construction européenne, qu'incarnent en particulier les droites radicales populistes. L'analyse de ce succès suggère la conjonction d'un vote protestataire à l'échelle nationale sur fond de crise économique, et d'un rejet croissant de l'Union, notamment à l'ouest de l'Europe. Les enjeux européens ont occupé une place centrale dans l'offre programmatique des principaux acteurs de la droite radicale populiste européenne. Ces derniers ont articulé un discours protectionniste et anti-immigration, assorti d'une critique forte des plans d'austérité et de sauvetage de l'Euro, consolidant leur base électorale populaire. L'analyse de leurs performances montre cependant d'importantes variations, qui témoignent de la fragmentation de cette famille politique et de son incapacité à se constituer en force organisée au sein du Parlement européen.

\footnotetext{
Abstract

The 2014 European elections have shown a clear rise in support for Eurosceptic parties. Euroscepticism is however a heterogeneous phenomenon combining 'soft' mainstream Euro-realism with 'hard' types of opposition to European integration, as revealed in particular in the competitive positions taken by populist
}

33. Sur la période 1979-2014, le score obtenu par ces partis aux scrutins européens est positivement corrélé avec leurs performances aux élections générales qui suivent directement (corrélation significative de 0,85 $\mathrm{N}=89$ ). Cette corrélation témoigne de la faculté de ces partis de capitaliser sur d'éventuels succès aux Européennes dans l'arène politique nationale. 
radical right parties. These parties have mobilized both on national anti-incumbent protest fed by popular discontent with the economic crisis, and on growing dissatisfaction with the EU, in particular in West European member states. European issues were central to the radical right's programmatic supply composing a mix of economically protectionist and anti-immigration policies, together with a strong criticism of austerity and EU bailout plans, which helped those parties consolidate electoral support among the lower social strata. Analyzing their performances across the EU shows a great deal of variation however, which attests to the political fragmentation of the populist radical right party family, accounting also for their failure to build stable transnational alliances. 